\title{
Coincident Patterns of Suicide Risk Among Adult Patients with a Primary Solid Tumor: A Large-Scale Population Study
}

This article was published in the following Dove Press journal: International Journal of General Medicine

\author{
Wen $\mathrm{Ma}^{1,2, *}$ \\ Wentao $\mathrm{Wu}^{2}$,* \\ Rong $\mathrm{Fu}^{2}$ \\ Shuai Zheng ${ }^{3}$ \\ Ruhai Bai (D) ${ }^{2}$ \\ Jun Lyu $\mathbb{D}^{1-3}$
}

'Clinical Research Center, The First Affiliated Hospital of Xi'an Jiaotong University, Xi'an, Shaanxi, People's Republic of China; ${ }^{2}$ School of Public Health, Xi'an Jiaotong University Health Science Center, Xi'an, Shaanxi, People's Republic of China; ${ }^{3}$ Department of Clinical Research, The First Affiliated Hospital of Jinan University, Guangzhou, Guangdong, People's Republic of China

*These authors contributed equally to this work
Correspondence: Jun Lyu

Clinical Research Center, The First

Affiliated Hospital of Xi'an Jiaotong

University, Xi'an, Shaanxi, 71006I,

People's Republic of China

Tel +86 13379060167

Email lyujun2020@jnu.edu.cn
Background: Suicide rate is much higher in cancer patients than in general population. This study examined the suicide risk in survivors of primary solid tumor across 19 cancer sites considering risk coincident patterns based on area-based SES indicators.

Methods: A retrospective search of the SEER database was conducted. Independent risk factors for suicide were identified using the Cox proportional-hazards model. Exploratory factor analysis and cluster analysis were used to create coincident patterns of SES factors.

Results: Suicide risk was higher for patients with a primary solid tumor who were older, male, white, unmarried, had no insurance, poorly differentiated, distant metastasis and did not undergo active treatment (especially surgery). The suicide risk was higher for patients living in areas with economic and education disadvantage, high levels of immigration and crowding, and high levels of residential instability. Concomitant presence of high economic and education disadvantage, high immigration and crowding levels and low residential instability, showed the highest risk of suicide.

Conclusion: In order to mitigate suicidal risk, clinicians should pay more attention to patients who are older, male, white, not married, high levels of cancer severity, not received active treatment (especially surgery), and having no insurance. Identifying coincident patterns of suicide help further screen high suicidal risk patients based on area-based socioeconomic status.

Keywords: socioeconomic status, suicide risk, solid tumor, exploratory factor analysis, cluster analysis, coincident patterns

\section{Introduction}

Cancer and suicide are both major causes of death worldwide, ${ }^{1}$ and patients with cancer have a suicide rate that is double that of the general population. ${ }^{2,3}$ Cancer affects patients in many ways, including increasing psychological distress, worsening psychosocial conditions, and decreasing the quality of life. Many of these factors are related to suicide risk. ${ }^{4-6}$

Similar to how unmanaged distress is associated with suicidal behavior in the general population, depressive disorders (including major and minor depression, and demoralization) affect up to $60 \%$ of patients with cancer. ${ }^{7}$ The side effects of cancer treatment, such as fatigue and pain, also cause a depressive mood, which is difficult to distinguish from cancer-related depression. ${ }^{8}$ Identifying patients with a high risk of suicide is vital for maintaining ongoing increases in cancer survival rates. 
Previous studies have reported that patients across several cancer sites have an increased risk of suicidal death. ${ }^{9-12}$ Zaorsky NG has identified specific suicide risk factors in US cancer patients, including male gender, advanced disease, white race, and unmarried status. ${ }^{13}$ In patients with hematological cancers, higher suicide risk was found in those without active treatment. ${ }^{14}$ However, few studies were found to investigate suicide risk factors in whole patient population with a primary solid tumor. Cancer is commonly divided into solid cancers and hematological cancers, and solid tumors make up most of all cancers. Solid tumors share a similar staging method and cancer-directed surgery is the main therapy for solid tumors. Therefore, it is necessary to further elucidate underlying suicide risk factors in solid tumor patients.

Socioeconomic conditions play an important role in predicting suicide risk in the general population. ${ }^{15}$ Several studies have found an inverse relationship between the socioeconomic status (SES) and the incidence of suicide. The following socioeconomic indicators have been observed to determine this relationship: basic levels of education, ${ }^{16}$ employment in blue-collar occupations, ${ }^{17}$ and low income. ${ }^{18}$ However, more information is needed to identify the socioeconomic predictors for suicide risk among cancer patients.

This study, based on the Surveillance, Epidemiology, and End Results (SEER) database from the United States, analyzed the suicide risk among patients with a primary solid tumor. The aim of this study is to determine the suicide risk among patients with a primary solid tumor while identifying coincident patterns with high suicide risk according to the area-based socioeconomic status.

\section{Materials and Methods Data Source}

This was a retrospective study based on the 18-registry SEER database (with additional treatment fields). Patients' data were retrieved using the SEER*Stat software (version 8.3.6). SEER is a publicly available, nationally representative, population-based cancer database which contains more than 8 million cancer cases. SEER database spanned 4 decades and cover $28 \%$ of the United States population, which is considered a valid source of cancer incidence and survival data in the United States. ${ }^{19-21}$ SEER has developed and maintained high-quality, validated data on causes of death among cancer survivors, providing insight into relative and cause-specific deaths in this population.
Publicly available de-identified data from the SEER database were exempt from requiring a medical ethics review, and informed consent was not required.

\section{Selection of Patients}

The suicide risk of patients diagnosed with a primary solid tumor between 1975 and 2016 was analyzed. The following 19 most-common sites for primary solid tumors in US patients were analyzed: brain, breast, cervix uteri, colorectum, esophagus, gallbladder, kidney, larynx, liver, lung and bronchus, skin (melanoma), oral cavity, ovary, pancreas, prostate, small intestine, stomach, thyroid, and urinary bladder. Patients who were younger than 18 years, diagnosed via autopsy or death certificate, or whose detailed information was not available were not included in this study.

\section{Variable Selection}

The following data were collected from the SEER database: site of cancer, age at diagnosis, sex, race, marital status, grade, stage, year of diagnosis, insurance status, therapy methods, cause of death, and survival time. After excluding patients younger than 18 years old, age groups were classified by every 20 years (18-39, 40-59, 60-79, and $\geq 80$ years old). Sex was classified as male or female. Race was classified as black, white, or other. Grade was classified as Grade1 (well differentiated), Grade2 (moderately differentiated), Grade3 (poorly differentiated), Grade4 (undifferentiated). Stage was classified as localized, regional, distant. Marriage was classified as married, unmarried (including divorced, separated, or widowed), or never married (single or domestic partner). Insurance status was classified as insured (including Medicare or Medicaid) or uninsured. The included therapy methods were surgery, radiation, and chemotherapy. Year of diagnosis was grouped by every ten years from 1975 to 2016.

The crude and age adjusted suicide rate from 1975 to 2107 was extracted from SEER database Rate Session. The Rate was displayed as cases per 100000 and the standard population was 2000 US std Population (19 age groups - Census P25-1130).

\section{Outcome Definition}

Mortality codes in SEER are assigned from death certificates, completed by the doctor caring for the patient at the time of demise. Patients were considered to have committed suicide if the cause of death was coded as: suicide and self-inflicted injury $(50,220)$. Survival time in SEER is 
measured in months, and the minimum time is 1 month to any event. Living patients were censored at the time when the data were collected.

\section{Socioeconomic Status Indicators}

Socioeconomic status indicators are calculated using the Census American Community Survey (ACS) 5-year files. Various county-level SES indicators were examined. Education (3 variables): with an education of less than 9th grade, less than high school graduate, and at least a bachelor's degree. Poverty (4 variables): the percentage of persons below poverty, families below poverty, persons below $150 \%$ poverty, and persons below $200 \%$ poverty in the county of residence. Crowding ( 1 variable): the percentage of households with more than one person per room. Income and employment (3 variables): unemployment (the percentage of persons over age 16 years who were unemployed) and median family and median household income measured in 2013 inflation-adjusted dollars. Relocation ( 8 variables): the percentage of individuals in the county age 5 years old older and the percentage of individuals in the county age 1 year and older who remained in the same house, moved within the county, moved to a different county within the same state, and moved to a different state. Immigration (4 variables): the percentage of persons who were foreign born, percentage of language isolated and percentages (ages $\geq 1$ year) and (ages $\geq 5$ years) within the county that moved to the US from another nation.

\section{Statistical Analysis}

In this study, baseline clinicopathological features for patients who were or were not suicide decedents were evaluated and compared based on Chi-Squared test. A survival analysis was performed using the Cox proportional-hazards model to calculate hazard ratios, with the survival time being from diagnosis to suicide. Non-suicide deaths and living patients were censored. Twenty-three county-level socioeconomic variables available in the SEER database were subjected to an exploratory factor analysis using varimax rotation in order to reduce the number of variables. Each patient was assigned a factor score based on the result of exploratory factor analysis. A k-means clustering method was applied to these factor scores to form distinct groups within all patients based on heterogeneity of the SES factor structures. A univariate stratified Cox proportional-hazards model was used to determine how SES factors and SES cluster groups affect the suicide risk in cancer patients. All statistical tests were two sided, with statistical significance being determined by $\mathrm{P}<0$.05. All statistical analyses were performed using R software (www.r-project.org).

\section{Results}

\section{Characteristics of Patients}

This study reviewed 6,990,572 patients in the SEER database who had a primary solid tumor. The cause of death was suicide for $11,389(0.16 \%)$ of these patients. Patients who committed suicide were mostly male (83.2\%), white (92.4\%), and 60-79 years old at the time of their cancer diagnosis $(57.0 \%)$. Cancer patients were mostly diagnosed during year 2005 2016 (52.0\%), and the proportion of suicide patients became lower during the years of 2005 2016 (33.0\%) comparing to non-suicide patients counterparty $(52.1 \%)$. The cancer sites associated with the most suicide deaths were the prostate $(30.1 \%)$, lung/ bronchus $(13.0 \%)$, urinary bladder $(6.7 \%)$, and breast $(8.0 \%)$, which together constituted more than half of all cancer patients. Table 1 describes the baseline clinicopathological characteristics of the included patients.

\section{Suicide Risk According to Clinicopathological Characteristics}

Figure 1 shows the suicide risk for patients with a primary solid tumor as analyzed using the Cox proportionalhazards model. For most cancer sites, Patients who were older, male, white, and presenting more serious illness (Grade 3 and 4, Stage of Regional and Distant) were more likely to die by suicide. Patients who were married, had insurance, and undergone active treatment therapies (surgery radiation and chemotherapy) had a lower suicide risk.

There was a higher suicide risk for older patients with these cancer sites: kidney, lung/bronchus, skin (melanoma), thyroid, and urinary bladder, while younger patients with breast cancer indicated higher suicide risk. Radiation therapy was a protective factor for patients with breast, prostate, larynx, brain, or lung/bronchus cancer, while the suicide risk was increased in patients with colorectum cancer. Chemotherapy decreased the suicide risk in patients with breast, esophagus, prostate cancer.

Figure 1 also showed that the suicide risk significantly decreased during more recent years. Kaplan-Meier curve indicated a higher survival probability during the time interval of 1995 2016 and the trend of the curve did not 


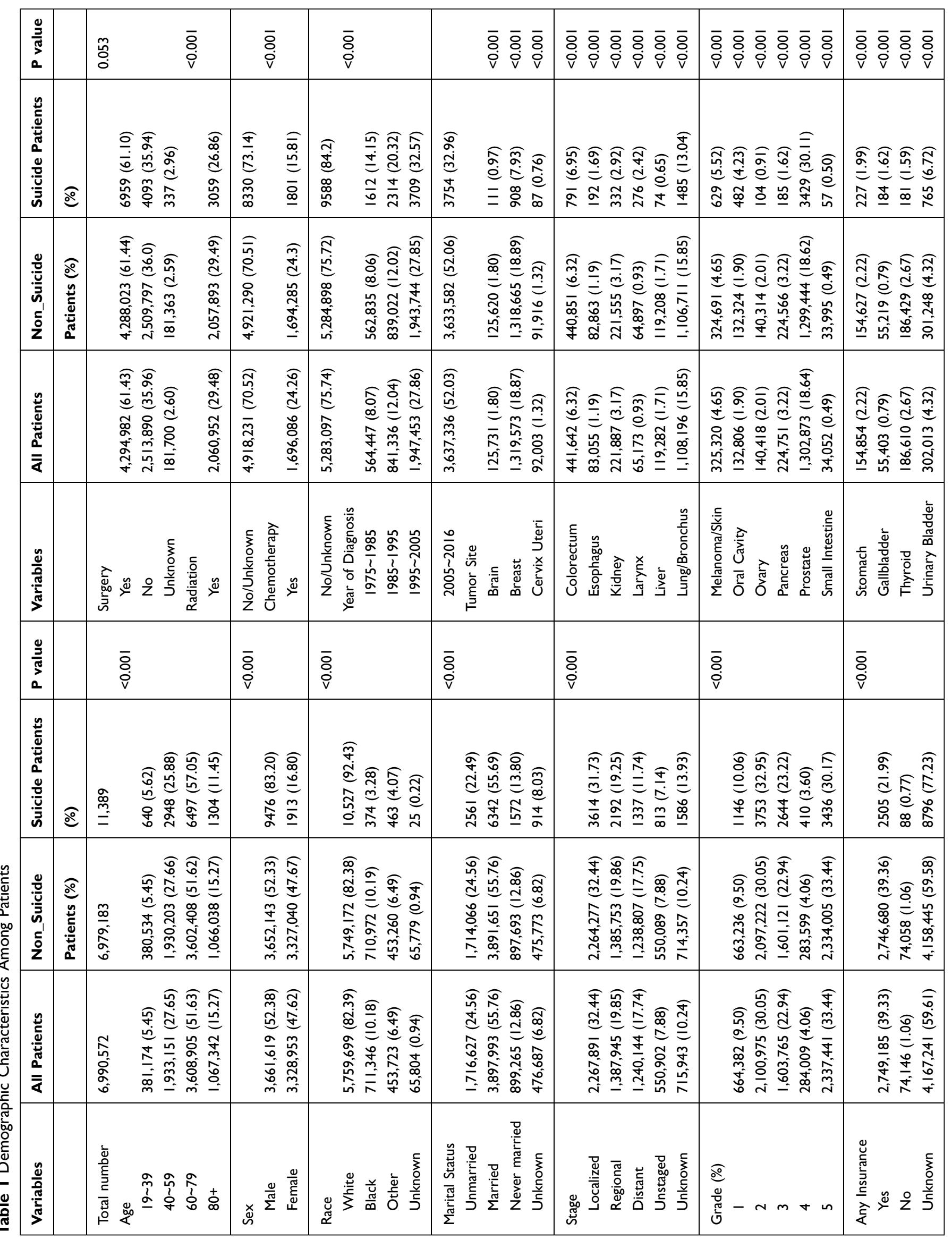




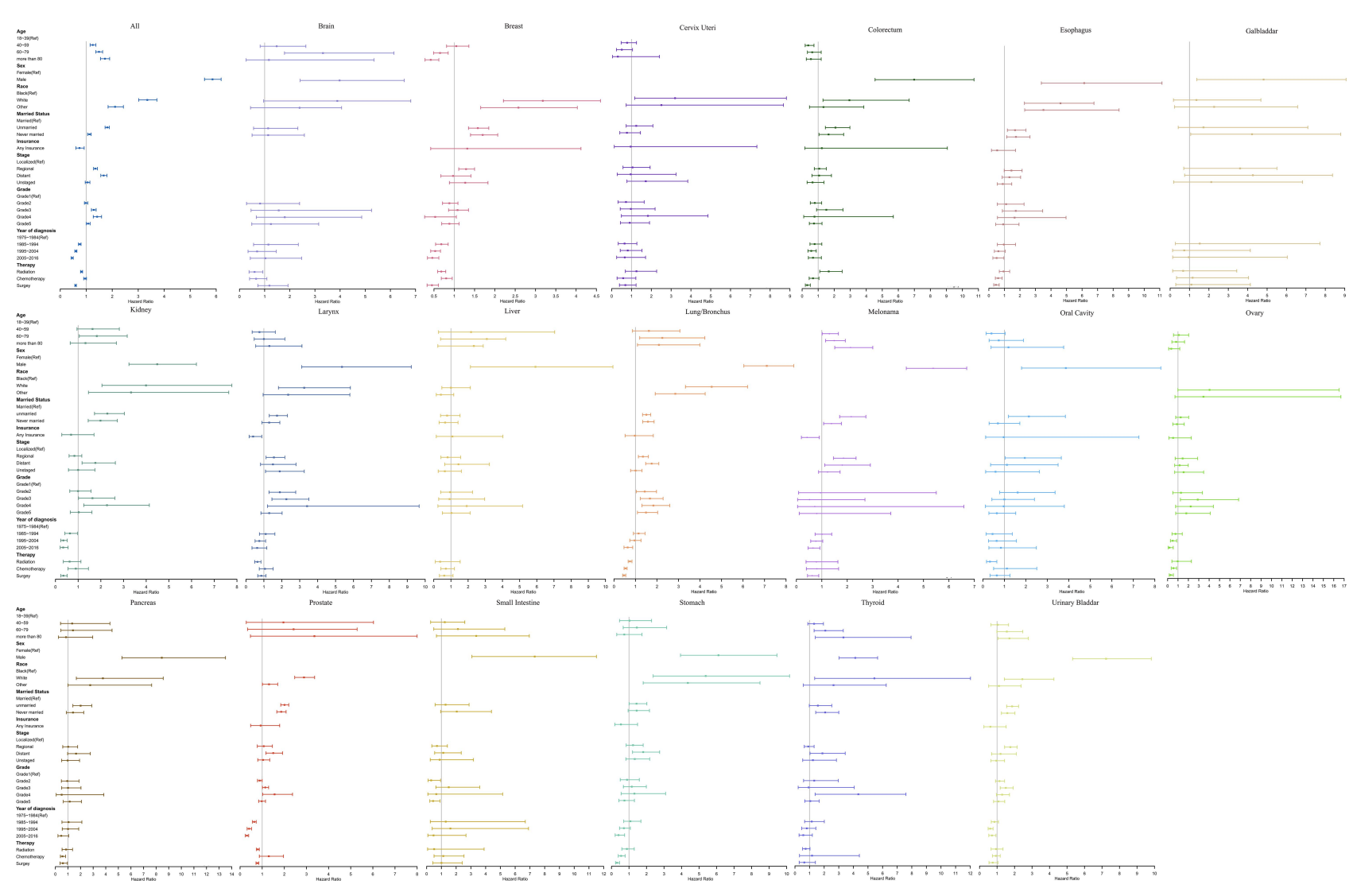

Figure I Impact of different clinical features on suicide risk by cancer sites.

Notes: Box indicated OR; segment indicated $95 \%$ confidence interval; OR smaller than I indicated benefit, and OR larger than I indicated hazard. Cases are limited to calculate OR of thyroid and colorectum under insurance, and OR of skin (melanoma) under race.

indicate an aggregation of suicide occurrence with diagnosis time (Supplementary Figure 1).

Further subgroup analysis, which divided patients into male group and female group, showed a similar suicide risk for different gender except for age and chemotherapy factors. Female patients aged 18-59 years (compared to more than 60 years) were exposed with higher suicide risk, while older male patients were more likely to die by suicide. The result of subgroup analysis is shown in Supplementary Figure 2.

\section{Trend in Suicide Rates, 1975-2017}

Figure 2 shows the trend of suicide rate (adjusted by age) in US across 19 sites of solid tumors. In general, suicide rate was much higher in male patients, and the suicide rate was decreasing both in men and women during the year from 1975 to 2017. For male patients, suicide rate was higher in cancer sites of prostate, lung/bronchus, Colorectum and Urinary Bladder (Figure 2A). For female patients, suicide rate kept at lower levels, and breast cancer accounted for a higher proportion of suicide risk
(Figure 2B). The crude suicide rate during the year 1975 to 2017 is shown in Supplementary Figure 3.

\section{Formation and Description of Socioeconomic Status Indicators: SES Factors and SES Clusters}

Factor analysis of the 23 SES variables resulted in a solution involving 4 factors that explained $100 \%$ of the variance in the original variables. Supplementary Figure 4 shows the correlation coefficients between the variables and factors (factor loading) and the proportion of information extracted from each variable. Factor 1 (the economic and education disadvantage factor) captured variables related to economic and education disadvantage, such as higher rates of community-level poverty, lower income, higher unemployment, a lack of higher education, and lower rates of immigration within the past year. Factor 2 (the residential instability factor) captured variables related to housing instability, such as lower rates of residents staying in the same house during the past year and higher 
A
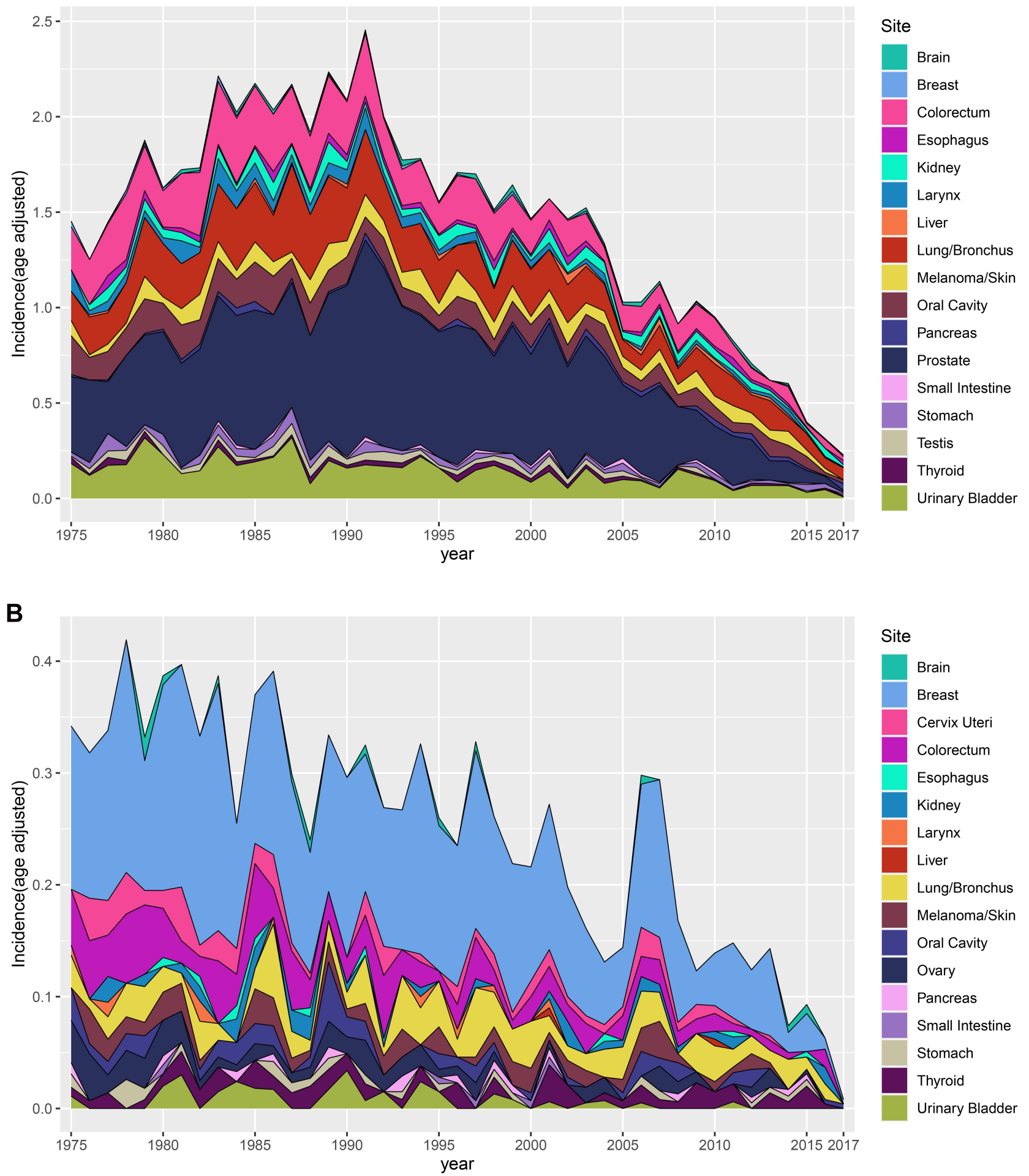

Figure 2 Trend in suicide rate for different gender, 1975-2017. (A) Suicide rate for male patients, (B) suicide rate for female patients. Note: Suicide rate was age-adjusted rate.

rates of them moving within counties. Factor 3 (the immigration and crowding factor) captured variables related to immigration, such as higher rates of community-level language isolation, not being born in the US, crowding, education at or below 9th grade, and immigration to the US within the past year.

Cluster analysis of the SES factor scores identified four distinct SES groups. Figure 3A illustrates the 


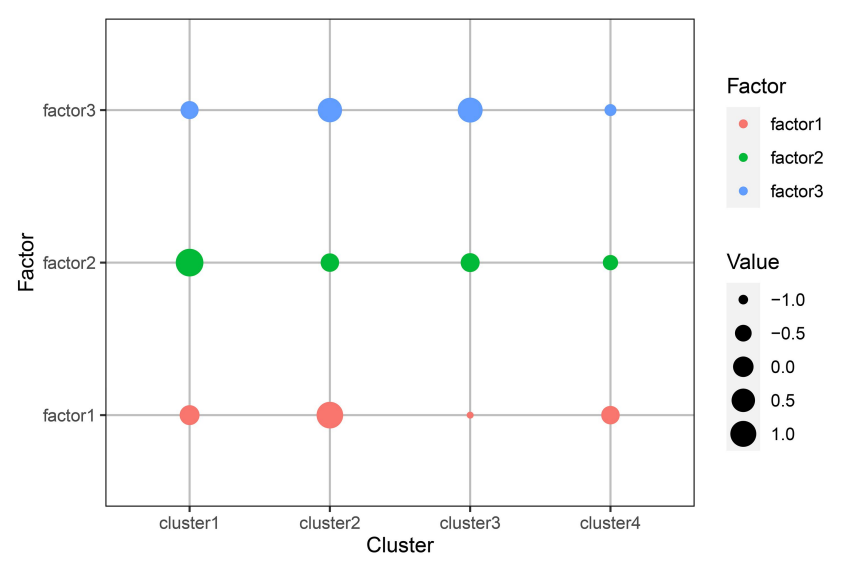

A

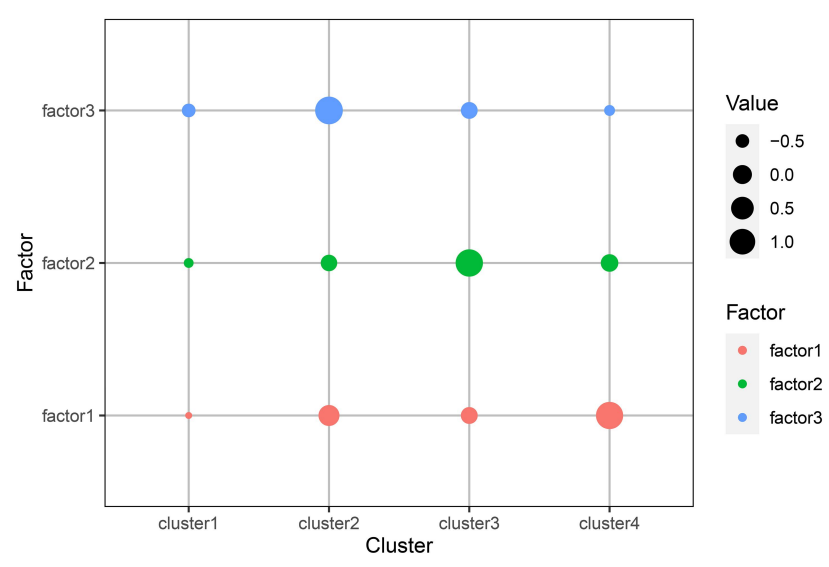

B

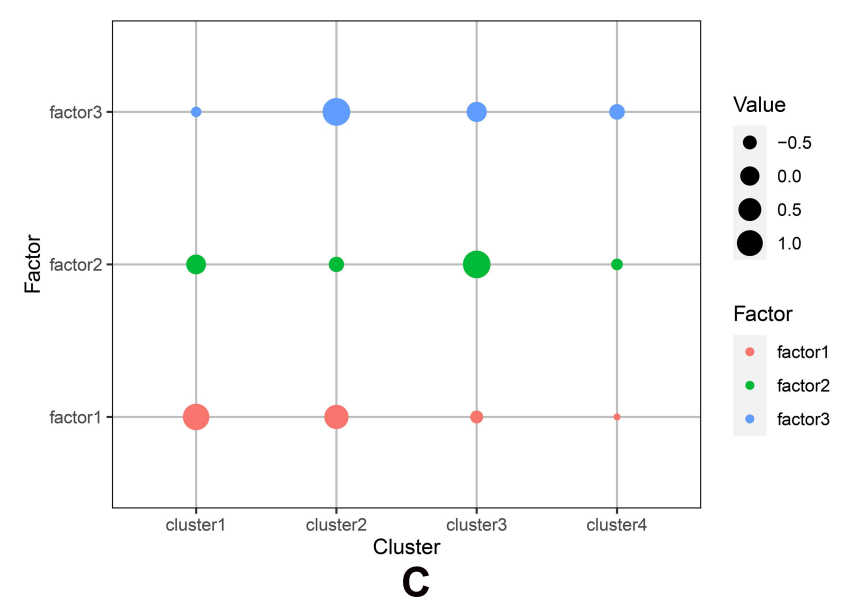

Figure 3 Patterns of SES factor scores across SES clusters. (A) All patient pattern, (B) male patient pattern, (C) female patient pattern.

Notes: Factor I (economic and education disadvantage): higher rates of community-level poverty, lower income, higher unemployment, a lack of higher education, and lower rates of immigration within the past year. Factor 2 (residential instability): lower rates of residents staying in the same house during the past year and higher rates of them moving within counties. Factor 3 (immigration/crowding): higher rates of community-level language isolation, not being born in the US, crowding, education at or below 9th grade, and immigration to the US within the past year.

patterns of factor scores across the clusters, while Table 2 presents the county-level socioeconomic characteristics of patients with a primary solid tumor falling into each of the four cluster groups. Cluster 1 is distinguished by medium economic and education disadvantage factors (Factor 1), high residential instability (Factor 2) and medium levels of immigration and crowding (Factor 3 ). Cluster 2 is characterized by high levels of economic and education disadvantage (Factor 1), low levels of residential instability (Factor 2), and high levels of immigration and crowding (Factor 3). Cluster 3 is distinguished by the high proportion of immigration and crowding (Factor 3), moderate levels of residential instability factors (Factor 2) and lowest levels of economic and education disadvantage (Factor 1). Cluster 4 is distinguished by all SES factors being low.
Factor analysis was used to concentrate SES factors in male and female subgroups and the four identical factors were extracted. Figure $3 \mathrm{~B}$ and $\mathrm{C}$ illustrate the patterns of factor scores across the clusters in male and female patients. For male, Cluster 1 is distinguished by all SES factors being low. Cluster 2 has highest immigration and crowding (Factor 3), medium rates of other two factors. Cluster 3 is distinguished by high levels of residential instability (Factor 2), factors related to low levels of other two factors. Cluster 4 is distinguished by high levels of economic and education disadvantage (Factor 1) and low levels of other two factors. For female patients, Cluster 1 is distinguished by highest economic and education disadvantage factors (Factor 1), factors related to medium levels of residential instability (Factor 2), and lowest levels of immigration and crowding (Factor 3). Cluster 2 is distinguished by high 
economic and education disadvantage factors (Factor 1) and immigration and crowding (Factor 3), low rate of residential instability (Factor 2). Cluster 3 is distinguished by highest levels of residential instability (Factor 2), medium immigration and crowding levels (Factor 3) and low economic and education disadvantage (Factor 2). Cluster 4 has low levels of all factors. Supplementary Tables 1 and 2 present the statistics of area-based SES factors under the different clusters among cases in different gender.

\section{Identifying the Suicide Risk Factors and Groups}

The suicide risk associated with each SES factor and cluster was analyzed using the Cox proportional-hazards model. Factor 1 and Factor 3 were adverse factors for the suicide risk, while Factor 2 was a protective factor for suicide (after adjusting for age, race, sex and insurance status) (Figure 4A). Figure 4B shows the highest risk cluster: patients in areas with high economic and education disadvantage, high immigration and crowding levels, low level of residential instability were identified as having higher suicide risk (Cluster 3) (OR=1.13, 95\% $\mathrm{CI}=1.11-1.45$ ) (after adjusting for age, race, sex and insurance status).

In male and female patient subgroup, Factor 1 and Factor 3 were adverse factors for the suicide risk, while Factor 2 was a protective factor for suicide (Supplementary Figure 5). This result was similar to whole patient analysis. Male patients in areas with

Table 2 Characterization of Socioeconomic Variables by Clusters

\begin{tabular}{|c|c|c|c|c|}
\hline & Cluster I Mean (SD) & Cluster2 Mean (SD) & Cluster3 Mean (SD) & Cluster4 Mean (SD) \\
\hline \multicolumn{5}{|c|}{ Economic and educational disadvantage factor } \\
\hline$\%<$ High School & $36.21(9.39)$ & $24.11(6.89)$ & $43.97(7.06)$ & $30.36(9.79)$ \\
\hline$\%$ At least Bachelors & $10.03(3.07)$ & $20.13(4.35)$ & $11.66(2.50)$ & 9.89 (3.49) \\
\hline \% Families below poverty & $9.84(3.4 I)$ & $15.68(3.78)$ & $6.70(1.64)$ & $8.27(3.00)$ \\
\hline$\%$ Persons below poverty & $14.32(4.23)$ & $19.95(4.34)$ & $10.00(2.08)$ & $11.52(3.66)$ \\
\hline$\%$ Persons $<150 \%$ of poverty & $22.74(5.89)$ & $31.37(5.14)$ & $16.45(3.05)$ & $19.20(5.74)$ \\
\hline$\%$ Persons $<200 \%$ of poverty & $31.28(7.12)$ & $41.64(5.52)$ & $23.20(3.89)$ & $27.29(7.44)$ \\
\hline$\%$ Unemployed & $4.06(1.81)$ & $10.10(3.84)$ & $6.42(1.70)$ & $3.68(1.60)$ \\
\hline Median household income & $6311.64(1259.23)$ & $5252.59(998.44)$ & $8888.98(1079.95)$ & $6441.88(1380.38)$ \\
\hline Median family income & $7774.62(1504.68)$ & $6134.01(932.89)$ & $10,519.18(1416.86)$ & $7996.28(1677.14)$ \\
\hline \multicolumn{5}{|c|}{ Immigration + Crowding factor } \\
\hline$\%<9$ th Grade & $6.47(1.58)$ & $9.18(2.01)$ & $5.96(0.95)$ & $6.17(1.67)$ \\
\hline \% Crowding & $3.21(1.69)$ & $7.20(4.00)$ & $6.24(2.37)$ & $1.86(1.00)$ \\
\hline \% Foreign born & $12.93(6.77)$ & $20.82(12.10)$ & $28.71(6.73)$ & $8.86(5.50)$ \\
\hline \% Language isolation & $3.70(1.85)$ & $8.11(4.95)$ & $8.63(2.26)$ & $2.81(1.98)$ \\
\hline$\%$ Moved to US $(5+$ yrs $)$ & $0.87(0.46)$ & $0.56(0.22)$ & $1.15(0.36)$ & $0.45(0.26)$ \\
\hline \% Moved to US $(I+y r s)$ & $0.88(0.46)$ & $0.56(0.22)$ & $1.16(0.36)$ & $0.45(0.27)$ \\
\hline \multicolumn{5}{|l|}{ Residential instability factor } \\
\hline \multicolumn{5}{|l|}{ Age $5+$ years } \\
\hline$\%$ Moved within county & $10.4 \mathrm{I}(\mathrm{I} .5 \mathrm{I})$ & $8.45(1.62)$ & $6.96(1.27)$ & $6.44(1.54)$ \\
\hline$\%$ Moved across state & $3.16(1.17)$ & $\mathrm{I} .17(0.45)$ & $\mathrm{I} .88(0.83)$ & $\mathrm{I} .83(0.78)$ \\
\hline$\%$ No moving & $82.12(2.56)$ & $87.38(2.08)$ & $87.33(1.65)$ & $88.09(2.09)$ \\
\hline \multicolumn{5}{|l|}{ Age I+ years } \\
\hline$\%$ Moved within county & $10.59(1.49)$ & $8.70(1.67)$ & $7.09(1.30)$ & $6.62(1.58)$ \\
\hline$\%$ Moved across state & $3.19(1.19)$ & I.I8 (0.47) & $1.90(0.86)$ & $\mathrm{I} .87(0.79)$ \\
\hline$\%$ No moving & $81.89(2.52)$ & $87.11(2.17)$ & $87.17(1.62)$ & $87.82(2.12)$ \\
\hline
\end{tabular}

Notes: All SES variables with percentage of persons living within a county with that characteristics calculated by Census American Community Survey (ACS) 2013-2017; median family income and median household income are in dollars; ANOVA $p$ value $<0.05$ for all variables. 


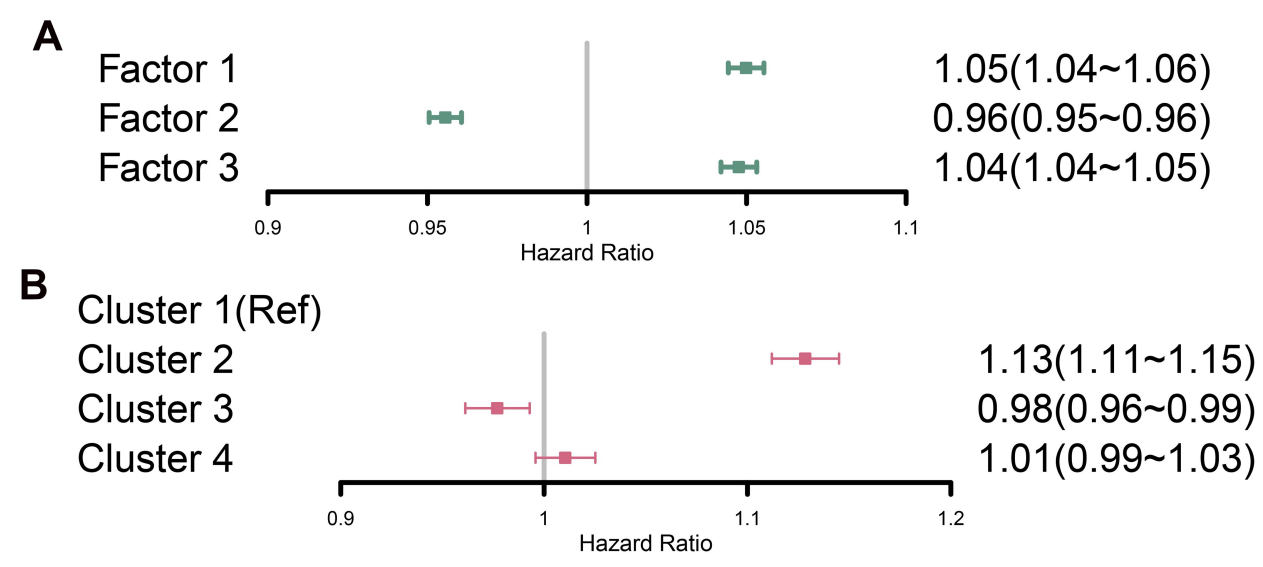

Figure 4 Adjusted hazard risk of primary solid tumor associated with socioeconomic factors (A) and clusters (B).

Notes: Adjusted models controlled for the following prognostic indicators: age at diagnosis, race, sex and insurance status.

medium economic and education disadvantage, medium residential instability, and high immigration and crowding levels were identified as having a higher suicide risk $(\mathrm{OR}=1.14$, 95\% $\mathrm{CI}=1.12-1.16$ ) (Figure 5A). For female patients, living in areas with medium levels of economic and education disadvantage, high immigration and crowding levels and low residential instability were shown a higher suicide risk $(\mathrm{OR}=1.21,95 \% \quad \mathrm{CI}=1.18-1.24)$ (Figure 5B).

\section{Discussion}

Suicide is a complex phenomenon that has significant sociological and psychiatric implications. This study focused on the risk of suicide among 6.99 million patients with a primary solid tumor, and analyzed the impacts of clinical and socioeconomic features. Although the occurrence of suicide is difficult to predict, our study indicated that patients who are older, male, white, not married, high levels of cancer severity, have not received active treatment (especially surgery), and have no insurance have a higher risk of suicide.

Our findings for patients with a primary solid tumor appear to be consistent with reports related to other types of cancer. Previous studies have shown that older cancer patients and members of the general population are more likely to commit suicide, ${ }^{10,22,23}$ which is consistent with the present findings. Older patients usually have a greater disease burden and social psychological pressures. Interestingly, younger and middle-aged patients with female genital system cancer (breast cancer, ovary cancer

\section{A \\ Cluster 1(Ref) Cluster2 Cluster 3 Cluster 4}

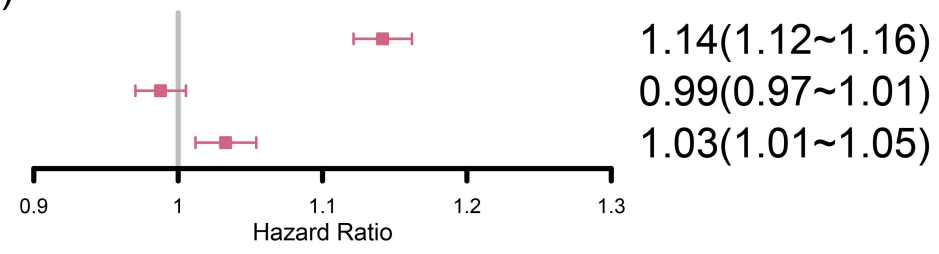

B

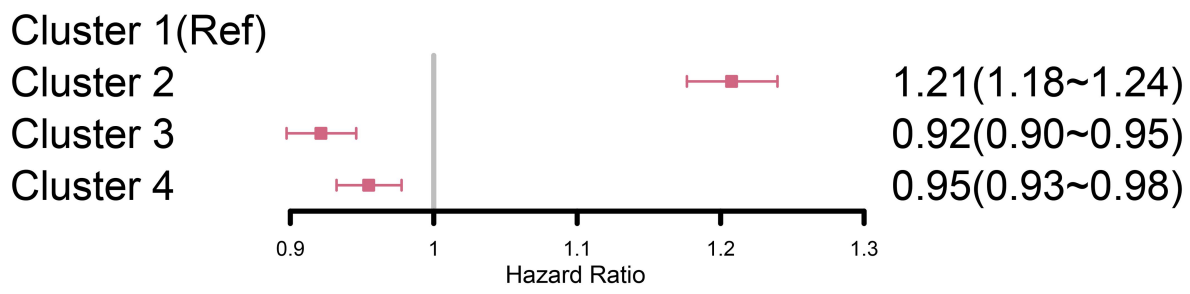

Figure 5 Adjusted hazard risk of primary solid tumor associated with socioeconomic clusters for different gender. (A) Adjusted hazard risk for male patients, (B) adjusted hazard risk for female patients.

Notes: Adjusted models controlled for the following prognostic indicators: age at diagnosis, race and insurance status. 
and cervix uteri) were found to have higher suicide risk, and the age difference of suicide risk among breast cancer patients was statistically significant. These findings were consistent with other studies. ${ }^{24,25}$ Studies reported that young women who developed invasive breast cancer experienced greater absolute and relative functional losses in physical role function, bodily pain, social function, and mental health, compared with middle-aged or elderly women with incident breast cancer. ${ }^{26}$ However, few studies further explained the pathological and psychological effect of the age difference for suicide risk in patients with ovary and cervix uteri cancer. This is possibly due to younger women suffered more from psychological burden losing sexual function and hormonal disorder from cancer and hormonotherapy. However, more studies are needed to explain these findings.

Being male was a risk factor for suicide in our study, which is consistent with the findings of other studies involving cancer patients. ${ }^{27}$ This is partially attributable to males being more likely to be aggressive and alcoholics, which is involved with mental disorder. The present study also corroborated the previous finding that race has a significant impact on suicide. The Centers for Disease Control and Prevention reported that the suicide rate in the US is lower in black people than in other races. ${ }^{28}$ The risk of dying from suicide was reportedly more than twice as high for white than for black patients with non-small-cell lung carcinoma. ${ }^{29}$ Our study similarly found a lower suicide risk in non-white patients for most cancer sites, which might be explained by their religious beliefs, family support, and culture of rejecting suicide. ${ }^{29}$

Providing social and medical support to patients with cancer plays a significant role in suicide prevention. We found that divorced or widow patients had a higher suicide risk, which might be due to them receiving less social support. ${ }^{30}$ Moreover, patients who had never married had a lower suicide risk than divorced or widow patients, which suggests that the latter patients tend to suffer more from a depressive mood.

The suicide risk was closely associated with the illness severity. In our study, patients with poorly differentiated and distant metastasis had higher suicide risk, which is due to their psychological pressure, fear of death and the pain of illness. Determining the optimal treatment options in cancer patients is complicated by treatments being affected by the illness severity. Few studies have systematically investigated the suicide risk in patients who have undergone chemotherapy and radiation therapy. Our study found that radiation and chemotherapy decreased suicide risk for many cancer sites. Nevertheless, cancer-related surgery was found to be a protective factor for almost all the cancer sites, which is consistent with previous findings. ${ }^{13,31-33}$ However, cognitive impairments of chemotherapy regimens were not distinguished in SEER database which may also induce suicidal ideation. ${ }^{34}$ For example, taxane-based chemotherapies confer risk for significant psychological symptoms, ${ }^{35}$ while some other drugs may only display mild or no symptoms of depression. Furthermore, patients with any type of insurance showed a lower suicide risk in our study, probably because the availability of insurance meant greater treatment support. Also, patients who can afford insurance tend to have a better economic status. Future developments in medical technology will result in treatment methods being more effective and efficient in alleviating the patients' suffering and improving prognoses.

Our study indicated that the age-adjusted rate for suicide was significantly decreased across almost all the solid tumor patients during the year from 1975 to 2017, which is consistent with other study. ${ }^{36}$ The improvement of living standard and medical technology may help decrease suicide risk for cancer patients. The enlargement of base number for cancer patients may also explain the decreasing suicide rate, since the development of cancer screening technology increased the diagnosis rate for cancer, especially in early stage. In our study, more than $50 \%$ of patients were diagnosed during the year from 2006 to 2016. However, studies also reported that the risk of suicide vs the general population is increasing: 1.9 in patients diagnosed 1973-2002, vs 4.4 for patients in the current work who were diagnosed 1973-2015. ${ }^{13,37}$ We did not discover the relationship between the suicide happening and the time of diagnosis from the aspect of total solid tumor patients. However, Nicholas G reported that certain cancer patients have higher standardized mortality rate (SMR) from suicide after the first year of diagnosis. ${ }^{13}$

When analyzing area-based SES factors, we used exploratory factor analysis to reduce the number of variables and empirically disentangle the complex underlying relationships between them, as well as to avoid the collinearity problem during modeling. The main principle underlying reducing the number of variables is based on the presence of strong correlations among similar factors.

The economic and education disadvantage factor (Factor 1), which corresponded to a socioeconomically 
vulnerable environment (lower education status, poverty, and unemployment), showed a higher suicide risk in cancer patients. Previous studies that investigated cancer patients and the general population support our result. ${ }^{38,39}$ Low-SES areas are often characterized by relatively few social control mechanisms, which may increase the likelihood of exposure to violence and feeling unsafe.

Previous studies have found lower suicide risks in immigration groups, which has been attributed to specific cultural/social aspects. ${ }^{40}$ This was not consistent with the effect of immigration/crowding (Factor 3) found in our study, possibly because the beneficial effects of pure immigration may be offset by other immigration-related aspects, since the immigration/crowding factor also included language isolation, crowding, and the lowest education levels. Patients living in areas with high level of residential instability (Factor 2) was a protective factor for suicide risk, which is consistent with the findings of studies of other population subgroups. ${ }^{41,42}$

Furthermore, cluster analysis was conducted with factor scores on these latent constructs with the aim of identifying distinct patterns of community-level SES indicators. This approach acknowledges that while these SES constructs often overlap, they may not all co-occur within specific communities, and their specific patterns of co-occurrence may indicate a higher suicide risk for certain cancer patients. We found that high-risk patients were clustered with these co-occurring features: high economic and education disadvantage, high immigration and crowding levels, and low residential instability (cluster 2). Interestingly, low residential instability was a protective factor analyzed alone, this was opposite in the result from cluster analysis. In real world, the coincidence of several risk factors presents accurate and true situation relative to single predictive factor analysis. One of the most important conclusions from our results is that interaction effects should be considered when analyzing the suicide risk in any population.

Subgroup analysis shows the similar characteristics of co-occurrence patterns with whole patient clusters. The high-risk pattern was clustered by factors with moderate/ high economic and education disadvantage, high immigration/crowding and low residential instability. However, studies reported that different social roles determine the ways that men and women are expected to behave differently ${ }^{43}$. Women are more likely to suffer from stressors experienced by others, ${ }^{44}$ so they suffer more living in areas with high immigration/crowding levels. Men are more likely to report financial stress, ${ }^{45}$ they are highly sensitive to economic and education disadvantage.

This study was subject to several limitations: (1) the inherent limitations of its retrospective design, (2) our inability to obtain SEER data on various potentially important factors, such as anxiety, depression, and pain, which are closely related to suicidal ideation, (3) treatment or medication effects, functional status, pain, disfigurement, and other important quality-of-life variables, are not available in SEER database, (4) depressive-effect of chemotherapy and radiation therapy were not distinguished in SEER database, (5) possible bias in the cause of death recorded in the database due to misclassification, (6) the results not including individuals who attempted suicide, and (7) the socioeconomic factors being based on an area-based evaluation (ie, county-based attributes) rather than the personalized socioeconomic status.

\section{Conclusion}

This study found that the risk of suicide death was significantly higher among adult patients with a primary solid tumor who were older, male, white, not received active treatment, had no insurance, high levels of cancer severity and were unmarried. The age-adjusted suicide rate has decreased during more recent years. Factor analysis indicated that more attention should be paid to patients living in areas of economic and education disadvantage, high levels of immigration/crowding and high levels of residential instability. Cluster analysis further helped to identify that a high suicide risk was associated with the concomitant presence of high economic and education disadvantage, high immigration and crowding levels and low residential instability.

\section{Ethics and Consent Statements}

Research involving human participants and/or animals: This article does not contain any studies with human participants or animals performed by any of the authors. Informed consent was waived as SEER is a de-identified, publicly available cancer database.

\section{Acknowledgment}

This study was supported by the National Social Science Foundation of China (No. 16BGL183).

\section{Author Contributions}

All authors made substantial contributions to conception and design, acquisition of data, or analysis and interpretation of data; took part in drafting the article or revising it critically for important intellectual content; agreed to 
submit to the current journal; gave final approval of the version to be published; and agree to be accountable for all aspects of the work.

\section{Disclosure}

The authors declare that they have no conflicts of interest for this work.

\section{References}

1. Fitzmaurice C, Allen C, Barber RM, et al. Global, regional, and national cancer incidence, mortality, years of life lost, years lived with disability, and disability-adjusted life-years for 32 cancer groups, 1990 to 2016: a systematic analysis for the global burden of disease study. JAMA Oncol. 2017;3:524-548. doi:10.1001/ jamaoncol.2016.5688

2. Fang F, Fall K, Mittleman MA, et al. Suicide and cardiovascular death after a cancer diagnosis. $N$ Engl J Med. 2012;366:1310-8.4. doi:10.1056/NEJMoa1110307

3. Anguiano L, Mayer DK, Piven ML, Rosenstein D. A literature review of suicide in cancer patients. Cancer Nurs. 2012;35:E14-26. doi:10.1097/NCC.0b013e31822fc76c

4. Pitman A, Suleman S, Hyde N, Hodgkiss A. Depression and anxiety in patients with cancer. BMJ. 2018;361:k1415. doi:10.1136/bmj. k1415

5. Fang C, Chang M, Chen P, et al. A correlational study of suicidal ideation with psychological distress, depression, and demoralization in patients with cancer. Support Care Cancer. 2014;22:3165-3174. doi:10.1007/s00520-014-2290-4

6. Tanriverdi D, Cuhadar D, Ciftci S. Does the impairment of functional life increase the probability of suicide in cancer patients? Asian Pac J Cancer Prev. 2014;15:9549-9553. doi:10.7314/ APJCP.2014.15.21.9549

7. Caruso R, GiuliaNanni M, Riba MB, Sabato S, Grassi L. Depressive spectrum disorders in cancer: diagnostic issues and intervention. A critical review. Curr Psychiatry Rep. 2017;19:33. doi:10.1007/ s11920-017-0785-7

8. Ullman K. Reducing risk of suicide in cancer patients. $J$ Natl Cancer Inst. 2017;109:3-5. doi:10.1093/jnci/djx025

9. Zachary K, Christopher JD, Thenappan C, et al. Cancer diagnosis and risk of suicide after accounting for prediagnosis psychiatric care: a matched-cohort study of patients with incident solid-organ malignancies. Cancer. 2019;125(16):2886-2895. doi:10.1002/ cncr.32146

10. Klaassen Z, Jen RP, DiBianco JM, et al. Factors associated with suicide in patients with genitourinary malignancies. Cancer. 2015;121:1864-1872. doi:10.1002/cncr.29274

11. Klaassen Z, Goldberg H, Chandrasekar T, et al. Changing trends for suicidal death in patients with bladder cancer: a $40+$ year population-level analysis. Clin Genitourin Cancer. 2018;16:206-212. doi:10.1016/j.clgc.2017.12.016

12. Saad AM, Gad MM, Al-Husseini MJ, et al. Suicidal death within a year of a cancer diagnosis: a population-based study. Cancer. 2019;125:972-979. doi:10.1002/cncr.31876

13. Zaorsky NG, Zhang Y, Tuanquin L, Bluethmann SM, Park HS, Chinchilli VM. Suicide among cancer patients. Nat Commun. 2019;10(1):207. doi:10.1038/s41467-018-08170-1

14. Hultcrantz M, Svensson T, Derolf $\AA$ R, et al. Incidence and risk factors for suicide and attempted suicide following a diagnosis of hematological malignancy. Cancer Med. 2015;4(1):147-154. doi:10.1002/cam4.316
15. Page A, Morrell S, Hobbs C, et al. Suicide in young adults: psychiatric and socio-economic factors from a case-control study. $B M C$ Psychiatry. 2014;14(1):68. doi:10.1186/1471-244X-14-68

16. Valkonen T, Martelin T. Occupational Class and Suicide: An Example of the Elaboration of a Relationship. Research Reports No.222, Department of Sociology. Helsinki: University of Helsinki; 1988.

17. Drever F, Bunting J. Patterns and trends in male mortality. In: Drever F, Whitehead M, editors. Health Inequalities. Decennial Supplement. London: Stationery Office; 1997.

18. Mortensen PB, Agerbo E, Erikson T, Qin P, Westergaard-Nielsen N. Psychiatric illness and risk factors for suicide in Denmark. Lancet. 2000;355:9-12. doi:10.1016/S0140-6736(99)06376-X

19. National Cancer Institute, Surveillance, Epidemiology, and End Results (SEER) Program. Overview of the SEER program. Bethesda, MD: National Cancer Institute; 2016. Available from: https://seer.cancer.gov/about/overview.html. Accessed February 28, 2018.

20. Howlader N, Ries LA, Mariotto AB, Reichman ME, Ruhl J, Cronin KA. Improved estimates of cancer-specific survival rates from population-based data. $J$ Natl Cancer Inst. 2010;102:1584-1598. doi:10.1093/jnci/djq366

21. Angela BM, Noone AM, Nadia H, et al. Cancer survival: an overview of measures, uses, and interpretation. $J$ Natl Cancer Inst Monogr. 2014;2014:145-186. doi:10.1093/jncimonographs/lgu024

22. Turaga KK, Malafa MP, Jacobsen PB, Schell MJ, Sarr MG. Suicide in patients with pancreatic cancer. Cancer. 2011;117:642-647. doi: $10.1002 /$ cncr. 25428

23. Kam D, Salib A, Gorgy G, et al. Incidence of suicide in patients with head and neck cancer. JAMA Otolaryngol Head Neck Surg. 2015;141:1075-1081. doi:10.1001/jamaoto.2015.2480

24. Apostolos G, Michail A, Michail P, Doreen W. Trends in incidence and associated risk factors of suicide mortality among breast cancer patients. Psycho Oncol. 2018;27(5):1450-1456. doi:10.1002/ pon. 4570

25. Caroline J, Violette AB, Rachel S, et al. Temporal trends and characteristics of suicide among women with gynecologic malignancy in the United States. Gynecol Oncol Rep. 2019;30:100510. doi:10.1016/ j.gore.2019.100510

26. Candyce HK, Bernard R, Chen WY, et al. Functional impact of breast cancer by age at diagnosis. J Clin Oncol. 2004;22(10):1849-1856. doi:10.1200/JCO.2004.04.173

27. Kendal W. Suicide and cancer: a gender-comparative study. Ann Oncol. 2006;18:381-387. doi:10.1093/annonc/mdl385

28. Xu J, Murphy SL, Kochanek KD, Bastian BA. Deaths: final data for 2013. Natl Vital Stat Rep. 2016;64:1-119.

29. Neeleman J, Wessely S, Lewis G. Suicide acceptability in Africanand white Americans: the role of religion. J Nerv Ment Dis. 1998;186 (1):12-16. doi:10.1097/00005053-199801000-00003

30. Jeong A, An J, Smith Fawzi MC. The moderating role of social support on depression and anxiety for gastric cancer patients and their family caregivers. PLoS One. 2017;12:e0189808. doi:10.1371/ journal.pone. 0189808

31. Clancy C, Burke JP, Barry M, Kalady MF, Calvin CJ. A metaanalysis to determine the effect of primary tumor resection for stage IV colorectal cancer with unresectable metastases on patient survival. Ann Surg Oncol. 2014;21(12):3900-3908. doi:10.1245/ s10434-014-3805-4

32. Ahmed S, Leis A, Fields A, et al. Survival impact of surgical resection of primary tumor in patients with stage IV colorectal cancer: results from a large population-based cohort study. Cancer. 2014;120 (5):683-691. doi:10.1002/cncr.28464

33. Samawi HH, Shaheen AA, Tang PA, Heng D, Cheung WY, Vickers MM. Risk and predictors of suicide in colorectal cancer patients: a surveillance, epidemiology, and end results analysis. Curr Oncol. 2017;24(6):e513-e517. doi:10.3747/co.24.3713 
34. Ahles TA, Saykin AJ. Candidate mechanisms for chemotherapy-induced cognitive changes. Nat Rev Cancer. 2007;7:192. doi:10.1038/nrc2073

35. Lisa MT, William EC, Charles LS, et al. Delayed emotional recovery after taxane-based chemotherapy. Cancer. 2008;113(3):638-647. doi:10.1002/cncr.23589

36. Jin Y, Gong HH, Siying C, et al. Incidence and risk factors for suicide death in male patients with genital-system cancer in the United States. Eur J Surg Oncol. 2019;45(10):1969-1976. doi:10.1016/j. ejso.2019.03.022

37. Misono S, Weiss NS, Fann JR, et al. Incidence of suicide in persons with cancer. J Clin Oncol. 2008;26:4731-4738. doi:10.1200/ JCO.2007.13.8941

38. Cairns JM, Graham E, Bambra C. Area-level socioeconomic disadvantage and suicidal behaviour in Europe: a systematic review. Soc Sci Med. 2017;192:102-111. doi:10.1016/j.socscimed.2017.09.034

39. Malenfant EC. Suicide in Canada's immigrant population. Health Rep. 2004;15(2):9-17.

40. Abdel-Rahman O. Socioeconomic predictors of suicide risk among cancer patients in the United States: a population-based study. Cancer Epidemiol. 2019;63:101601. doi:10.1016/j.canep.2019.101601
41. Glasheen C, Forman-Hoffman V, Hedden S, Ridenour T, Wang J, Porter J. Residential transience among US adolescents: association with depression and mental health treatment. Epidemiol Psychiatr Sci. 2019;28(6):682-691. doi:10.1017/S2045796018000823

42. Windfuhr K, While D, Hunt I. Suicide in juveniles and adolescents in the United Kingdom. J Child Psychol Psychiatry. 2008;49 (11):1155-1165. doi:10.1111/j.1469-7610.2008.01938.x

43. Canetto SS, Sakinofsky I. The gender paradox in suicide. Suicide Life Threat Behav. 1998;28(1):1-23.

44. De Vries B, Watt D. A lifetime of events: age and gender variations in the life story. Int J Aging Human Dev. 1996;42(2):81-102. doi:10.2190/FM22-V5VT-B60Y-6UGC

45. Vandello JA, Bosson JK, Cohen D, Burnaford RM, Weaver JR. Precarious manhood. J Pers Soc Psychol. 2008;95(6):1325. doi: $10.1037 / \mathrm{a} 0012453$
International Journal of General Medicine

\section{Publish your work in this journal}

The International Journal of General Medicine is an international, peer-reviewed open-access journal that focuses on general and internal medicine, pathogenesis, epidemiology, diagnosis, monitoring and treatment protocols. The journal is characterized by the rapid reporting of reviews, original research and clinical studies
Dovepress

across all disease areas. The manuscript management system is completely online and includes a very quick and fair peer-review system, which is all easy to use. Visit http://www.dovepress.com/ testimonials.php to read real quotes from published authors. 